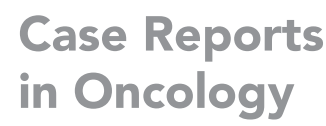

Case Reports
in Oncology

\author{
in Oncology
}

\title{
A Rare Case of Severe Lactic Acidosis from 5-Fluorouracil after mFOLFOX6 Treatment in a Patient with Advanced Gastric Cancer
}

\author{
Tae Hoon Lee ${ }^{a} \quad$ Dan Le ${ }^{a, b}$ \\ aFaculty of Medicine, University of British Columbia, Vancouver, BC, Canada; ${ }^{b}$ Division of \\ Medical Oncology, BC Cancer, Surrey, BC, Canada
}

Keywords

5-Fluorouracil $\cdot$ mFOLFOX6 $\cdot$ Lactic acidosis

\begin{abstract}
Gastric cancer is one of the most common cancers worldwide and is one of the deadliest types of neoplasm. Many patients present with an advanced stage where palliative chemotherapy is the standard of care. 5-Fluorouracil (5-FU) remains the backbone of systemic therapy treatment in advanced gastric cancer, although is associated with many side effects. While cases of encephalopathy caused by hyperammonemia have been reported, lactic acidosis after systemic 5-FU exposure is exceedingly rare. We present here for the first time a case of type $B$ lactic acidosis secondary to mFOLFOX6 therapy in advanced gastric cancer. This patient presented with acute delirium, dystonia, and a lactate of $11.7 \mathrm{mmol} / \mathrm{L}$, which peaked to 18.7 $\mathrm{mmol} / \mathrm{L}$, within $48 \mathrm{~h}$ of chemotherapy treatment. Routine clinical monitoring of lactate may be beneficial to avoid this potentially life-threatening adverse event.
\end{abstract}

(C) 2021 The Author(s).

Published by S. Karger AG, Basel

\section{Introduction}

Gastric cancer is one of the most common and deadliest forms of malignancy. It is the third leading cause of cancer deaths worldwide [1]. Despite improvements in early detection, many patients present with unresectable disease where palliative chemotherapy is the standard treatment [2]. Chemotherapy has been shown to improve survival and quality of life, and 5-fluorouracil (5-FU) in combination with oxaliplatin (FOLFOX) remains the main choice of treatment for patients with locally advanced unresectable or metastatic gastric cancer [3]. Common adverse effects associated with systemic exposure to 5-FU include hematologic, 

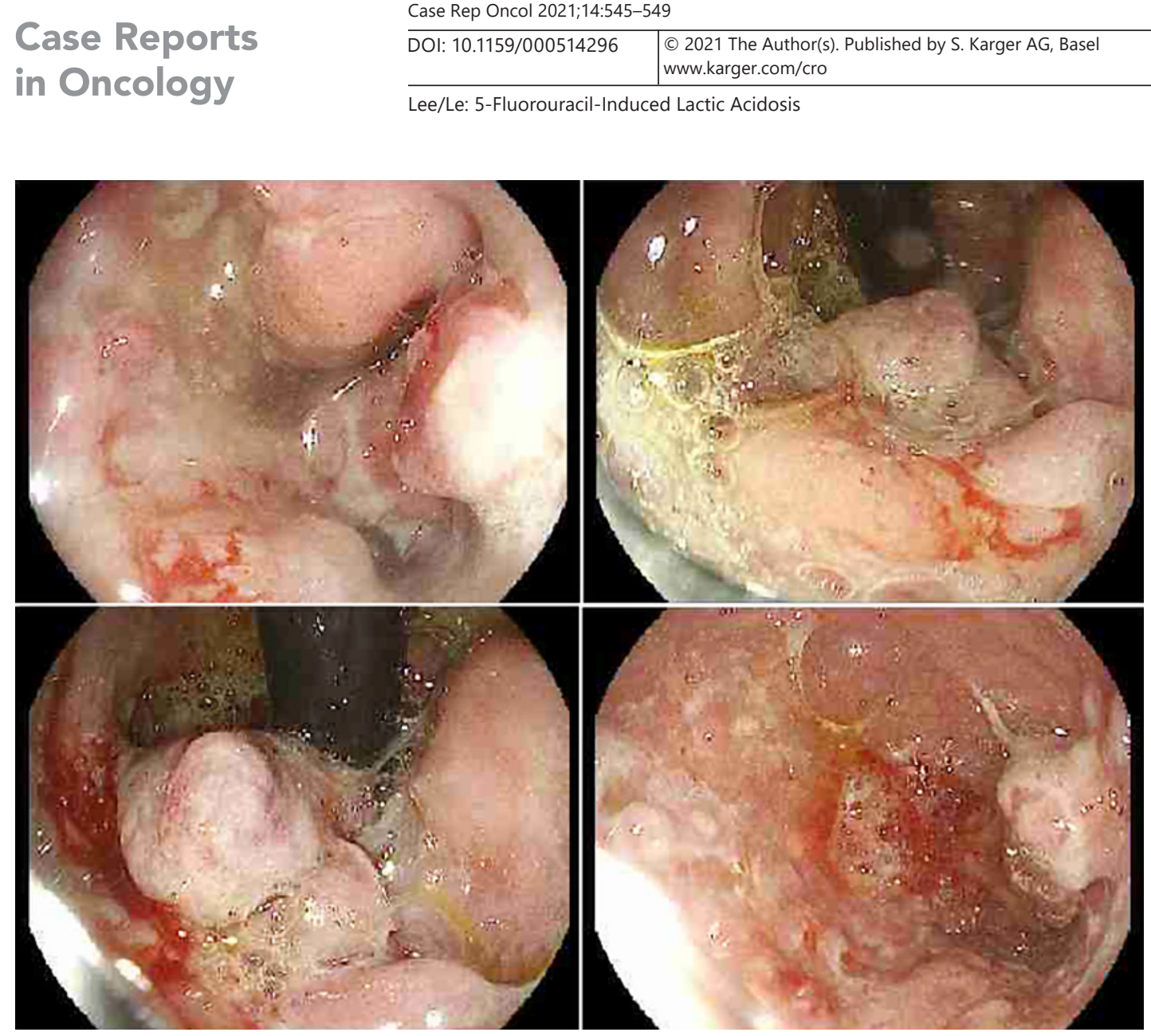

Fig. 1. Representative esophagogastroduodenoscopy images of near circumferential mass in the gastric body, fundus, and on the lesser curvature of the stomach.

gastrointestinal, dermatologic, and cardiovascular side effects [4]. While cases of encephalopathy caused by hyperammonemia have been reported, lactic acidosis associated with 5-FU-based chemotherapy is exceedingly rare [5-10]. Here, we present the first case of type $\mathrm{B}$ lactic acidosis secondary to systemic 5-FU treatment in advanced gastric cancer.

\section{Case Report}

A 56-year-old East Asian male was diagnosed with HER2-negative gastric adenocarcinoma by diagnostic upper endoscopy (Fig. 1). He was well until 3 months prior to this diagnosis, at which time he was also incidentally found to have poor renal function with an EGFR of $29 \mathrm{~mL} / \mathrm{min}$. A staging CT scan of the chest, abdomen, and pelvis showed no obvious evidence of metastatic disease, but there was minimal ascites and bilateral hydronephrosis with no clear etiology. He was seen by Urology, and bilateral nephrostomy tubes were inserted, resulting in an improvement in his hydronephrosis and renal function.

He had a diagnostic laparoscopy that confirmed malignant ascites on cytology. Given his advanced disease, he was initiated on palliative chemotherapy with mFOLFOX6. After cycle 2 , he was admitted for febrile neutropenia and completed a course of antibiotics for a Lactococcus garvieae urinary tract infection. His blood cultures were negative, and his lactate was $1.7 \mathrm{mmol} / \mathrm{L}$. Due to his febrile neutropenia, cycle 3 was dose reduced with the 5-FU bolus omitted. 
On day 3 of cycle 3 , he presented to the emergency room with acute delirium, dystonia, and an anion-gap metabolic acidosis with lactate of $11.7 \mathrm{mmol} / \mathrm{L}$, which increased to 18.7 $\mathrm{mmol} / \mathrm{L}$ on the same day. His symptoms started during the second half of the 46-h 5-FU continuous infusion as part of the mFOLFOX6 protocol. Vital signs at presentation included a blood pressure of $132 / 76$, heart rate of 80 , respiratory rate of $18, \mathrm{SpO}_{2}$ of $100 \%$ in room air, and a temperature of $36.4^{\circ} \mathrm{C}$. Other blood work included a white blood cell count of $14.8 \cdot 10^{9} / \mathrm{L}$, hemoglobin of $103 \mathrm{~g} / \mathrm{L}$, platelet count of $477 \cdot 10^{9} / \mathrm{L}$, sodium of $141 \mathrm{mmol} / \mathrm{L}$, potassium of 3.8 $\mathrm{mmol} / \mathrm{L}$, bicarbonate of $15 \mathrm{mmol} / \mathrm{L}$, creatinine of $157 \mathrm{mmol} / \mathrm{L}$, EGFR of $42 \mathrm{~mL} / \mathrm{min}$, anion gap of $24 \mathrm{mmol} / \mathrm{L}$, phosphate of $1.2 \mathrm{mmol} / \mathrm{L}$, and a magnesium of $0.9 \mathrm{mmol} / \mathrm{L}$. He had normal liver enzymes, bilirubin, and lipase. His CRP was $6.8 \mathrm{mg} / \mathrm{L}$, and CK was $80 \mathrm{U} / \mathrm{L}$. His betahydroxybutyrate and urine toxicology screen were negative. Venous blood gas showed a $\mathrm{pH}$ of 7.35 with a $\mathrm{CO}_{2}$ of $27 \mathrm{~mm} \mathrm{Hg}$ and bicarbonate of $15 \mathrm{mmol} / \mathrm{L}$. Blood and urine cultures were negative. CT scan of the head, abdomen and pelvis, and a chest X-ray showed no obvious etiology for his presentation. He was not on any medications or natural supplements other than the chemotherapeutic agents.

He was initiated on piperacillin-tazobactam and vancomycin as per sepsis protocol. The 5 -FU continuous infusion was disconnected on admission. He was then admitted to the High Acuity Unit for monitoring. Within $16 \mathrm{~h}$ of its peak level, his lactate normalized to $1.2 \mathrm{mmol} / \mathrm{L}$ with no further interventions. His delirium and dystonia also resolved. At the time of normalization of his lactate, his EGFR remained stable at $43 \mathrm{~mL} / \mathrm{min}$. He was discharged on the third day with a lactate of $0.8 \mathrm{mmol} / \mathrm{L}$.

His mFOLFOX6 therapy was discontinued and was switched to ramucirumab and paclitaxel. He responded to this treatment with CT evidence of a decrease in size of the gastric mass and no evidence of new metastatic disease after 4 cycles. However, midway through cycle 7, he was admitted to hospital with headache, neck stiffness, and nausea. He was diagnosed with leptomeningeal carcinomatosis with a lumbar puncture revealing malignant cells on cytology and an MRI of the head and spine showing leptomeningeal thickening and enhancement. He continued to decline despite being on high-dose steroids and was eventually transferred to hospice.

\section{Discussion}

Although 5-FU-based chemotherapy is associated with many well-known side effects [4], lactic acidosis during FOLFOX therapy is exceedingly rare with only 6 cases having been described in the literature among all cancer types [5-10]. None of these reported cases were within the context of gastric cancer. Lactic acidosis is defined as a serum lactate level greater than $4.0 \mathrm{mmol} / \mathrm{L}$ with a blood $\mathrm{pH}$ of 7.35 or lower. Typically, lactic acidosis is classified as type A or B depending on tissue hypoperfusion. This patient had a severe lactic acidosis of 11.7 $\mathrm{mmol} / \mathrm{L}$ on initial presentation, which peaked to $18.7 \mathrm{mmol} / \mathrm{L}$, that spontaneously normalized to $1.2 \mathrm{mmol} / \mathrm{L}$ within $24 \mathrm{~h}$. His negative sepsis workup and absence of tissue hypoperfusion suggests a type B lactic acidosis. Lactic acidosis due to gastric malignancy has been reported in the literature [11], but the acute and short presentation in this case makes this unlikely. Also, lactic acidosis has not been associated with oxaliplatin. A single case of renal tubular acidosis after oxaliplatin has been described, but this was in the context of normal lactate levels [12]. This patient presented during the 5-FU continuous infusion portion of his chemotherapy, making it the most likely causative agent, but its mechanisms are poorly understood.

Thiamine deficiency and hypotriglyceridemia have been proposed as mechanisms for 5-FU-induced lactic acidosis [5]. 5-FU increases thiamine metabolism inside cells, and this can lead to lactic acidosis because it prevents the oxidation of pyruvate into acetyl-CoA. Also, fluo-

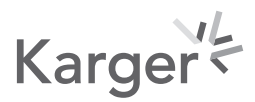


roacetate, which is an intermediate metabolite of $5-\mathrm{FU}$, has been shown to impair the tricarboxylic acid cycle [10]. Anaerobic respiration resulting from ATP-deprived state caused by fluoroacetate can be prolonged by hypotriglyceridemia, as it impairs the oxidation of fatty acids [5]. Thiamine and triglyceride levels were not measured, but this patient exhibited no signs of dry beriberi and had a good appetite, making their deficiency unlikely [6].

Ammonia is the final metabolite of 5-FU, and hyperammonemia has been associated with systemic 5-FU therapy in gastric cancer [13]. While cases of encephalopathy caused by hyperammonemia have been reported, there are only 4 cases of simultaneous presentation of lactic acidosis and hyperammonemia that have been reported in the literature $[5,6,9,10]$. Ammonium was not measured in this patient because he had unremarkable liver enzymes, but it may have contributed to his delirium and dystonia. On a similar note, it is unknown if this patient had dihydropyrimidine dehydrogenase (DPD). DPD is the enzyme responsible for breaking down 5-FU into 5-fluoro- $\beta$-alanine, carbon dioxide, and ammonia [14], and significant toxicities have been associated with rare familial deficiency of the enzyme [15]. Although DPD deficiency is unlikely to be the cause of lactic acidosis in this case given how well he tolerated the initial 2 cycles of treatment, it has not been completely ruled out.

Of note, this patient had poor renal function secondary to bilateral hydronephrosis with no clear etiology. Although the clearance of lactate is only minimally influenced by the kidneys, Yeung et al. [8] reported a case of type B lactic acidosis from 5-FU treatment in a colorectal cancer patient on hemodialysis. This raises the possibility that perhaps the mechanism driving type B lactic acidosis after 5-FU chemotherapy is influenced by renal function.

In conclusion, lactic acidosis is an extremely rare event associated with systemic 5-FU treatment. There are only 6 prior reports, and this is the first to identify this event in gastric cancer. Although the exact mechanisms are unclear, therapies involving 5-FU should be used with caution, and routine clinical monitoring with measurements of lactate may be beneficial within 24-48 h of bolus administration, particularly in patients with poor renal function.

\section{Statement of Ethics}

Written informed consent was obtained from the family of the deceased patient for publication of this case report and any accompanying images.

\section{Conflict of Interest Statement}

The authors have no conflicts of interest to declare.

\section{Funding Sources}

The authors received no financial support for this work.

\section{Author Contributions}

Tae Hoon Lee drafted the manuscript. Dan Le revised the manuscript. All authors read and approved the final manuscript.

\section{Karger'k}




\section{References}

1 Bray F, Ferlay J, Soerjomataram I, Siegel RL, Torre LA, Jemal A. Global cancer statistics 2018: GLOBOCAN estimates of incidence and mortality worldwide for 36 cancers in 185 countries. CA Cancer J Clin. 2018;68(6): 394-424.

2 Greenlee RT, Murray T, Bolden S, Wingo PA. Cancer statistics, 2000. CA Cancer J Clin. 2000;50(1):7-33.

3 De Vita F, Orditura M, Matano E, Bianco R, Carlomagno C, Infusino S, et al. A phase II study of biweekly oxaliplatin plus infusional 5-fluorouracil and folinic acid (FOLFOX-4) as first-line treatment of advanced gastric cancer patients. Br J Cancer. 2005;92(9):1644-9.

4 Papanastasopoulos P, Stebbing J. Molecular basis of 5-fluorouracil-related toxicity: lessons from clinical practice. Anticancer Res. 2014;34(4):1531-5.

5 Yeh $\mathrm{KH}$, Cheng AL. High-dose 5-fluorouracil infusional therapy is associated with hyperammonaemia, lactic acidosis and encephalopathy. Br J Cancer. 1997;75(3):464-5.

6 Rosen A, van Kuilenburg A, Assmann B, Kuhlen M, Borkhardt A. Severe encephalopathy, lactic acidosis, vegetative instability and neuropathy with 5-Fluorouracil treatment - pyrimidine degradation defect or beriberi? Case Rep Oncol. 2011;4(2):371-6.

7 Ito A, Kawamoto K, Park T, Ito T. [A case of mFOLFOX6-induced lactic acidosis in a patient with colon cancer]. Gan To Kagaku Ryoho. 2014;41(11):1445-7.

8 Yeung EK, Copland MA, Gill S. Type B lactic acidosis from fluorouracil in fluorouracil, oxaliplatin and leucovorin treatment for carcinoma of the colon in a hemodialysis patient. Clin Kidney J. 2018;11(6):786-7.

9 Hara Y, Miyamoto Y, Hiyoshi Y, Iwatsuki M, Baba Y, Yoshida N, et al. Severe encephalopathy, lactic acidosis and hyperammonaemia with FOLFIRI plus aflibercept after two-stage hepatectomy: a case report. In Vivo. 2019 Mar-Apr;33(2):563-5.

10 Fukuda M, Nabeta M, Muta T, Cho T, Shimamatsu Y, Shimotsuura Y, et al. Disturbance of consciousness due to hyperammonemia and lactic acidosis during mFOLFOX6 regimen: Case report. Medicine (Baltimore). 2020; 99(33):e21743.

11 Krimmel JD, Packer CD. Type B lactic acidosis in a patient with gastric adenocarcinoma and extensive hepatic metastases. Med Princ Pract. 2015;24(4):391-3.

12 Linch M, Cunningham D, Mingo O, Stiles A, Farquhar-Smith WP. Renal tubular acidosis due to oxaliplatin. Ann Oncol. 2007;18(4):805-6.

13 Chu G, Salzman J. Hyperammonemia after capecitabine associated with occult impairment of the urea cycle. Cancer Med. 2019;8(5):1996-2004.

14 Heggie GD, Sommadossi JP, Cross DS, Huster WJ, Diasio RB. Clinical pharmacokinetics of 5-fluorouracil and its metabolites in plasma, urine, and bile. Cancer Res. 1987;47(8):2203-6.

15 Tuchman M, Stoeckeler JS, Kiang DT, O'Dea RF, Ramnaraine ML, Mirkin BL. Familial pyrimidinemia and pyrimidinuria associated with severe fluorouracil toxicity. N Engl J Med. 1985;313(4):245-9. 